\title{
Análise do tema gestão estratégica nas pequenas empresas prestadoras de serviços: uma revisão bibliográfica
}

\author{
Andréa Simone Machiavelli Pontes ${ }^{1}$
}

RESUMO

As pequenas empresas prestadoras de serviço têm desempenhado cada vez mais um papel importantíssimo para o crescimento da economia brasileira. Tais empresas se deparam com novas situações de mercado como concorrência, excelência na qualidade, clientes exigentes e conhecedores, necessidade de abrangência no mercado, cultura e clima organizacional, fornecedores e outras tantas variáveis muitas vezes nem conhecidas por seus administradores. A abordagem da Gestão Estratégica apresenta a necessidade de entender as variáveis e fatores que compõem o micro e macro ambiente das pequenas empresas, o processo de planejamento e de ações estratégicas que conduzam os objetivos e metas contribuindo para o sucesso das empresas diante de um mercado competitivo. 0 objetivo deste artigo é realizar uma revisão bibliográfica em torno do conceito Gestão Estratégica, para identificar como as pequenas empresas prestadoras de serviços implementam ações para suportar a ação estratégica. 0 método de pesquisa foi uma análise bibliométrica, por meio de procedimentos de pesquisa quantitativos e qualitativos, com análise documental de publicações no período entre 2006 e 2012, buscando-se artigos que contivessem em seus títulos o termo "Gestão Estratégica" com base de dados da biblioteca eletrônica SciELO.

Palavras-chave: Gestão Estratégica. Pequenas empresas. Revisão Bibliográfica.

\section{INTRODUÇÃO}

Considerando que as grandes mudanças tecnológicas, sociais, culturais, demográficas ou legais que ocorrem no mundo levam as pequenas empresas prestadoras de serviços a buscarem novas possibilidades de crescimento, desenvolvimento ou manutenção de sua posição no mercado, torna-se essencial a busca de informações consistentes que demonstrem por meio de pesquisas e experiências a importância da Gestão Estratégica. 
As pequenas empresas prestadoras de serviço integrantes de um mercado competitivo devem perceber o envolvimento cada vez mais relevante da estratégia nas suas gestões. De acordo com Almeida (2011), o Planejamento Estratégico, quando aplicado às pequenas empresas, necessita de um grande grau de simplificação e enfrenta a dificuldade de conseguir que o empresário saia da rotina, sendo necessário criar um comprometimento com a sua equipe estimulando o desenvolvimento do trabalho.

Conhecer os principais obstáculos, saber avaliar as condições estratégicas, como tratar riscos e turbulências, assim como perceber e aproveitar as oportunidades estratégicas são procedimentos fundamentais para que as empresas possam formular ações sustentáveis, construindo caminhos e alternativas para implementar suas estratégias competitivas.

A Gestão Estratégica confronta-se com tal necessidade, visando, por intermédio do planejamento estratégico, da organização de ações estratégicas e do diagnóstico estratégico, demonstrar o que está acontecendo e o que poderá ocorrer. A Gestão Estratégica possibilita a projeção de situações futuras que auxiliarão as empresas na definição de seus objetivos estratégicos, no levantamento de alternativas condizentes e na decisão estratégica eficaz.

Nesse sentido, este estudo delimita a sua problemática qual seja: Como as pequenas empresas prestadoras de serviços poderão implementar ações que possam suportar a Gestão Estratégica, baseadas na literatura sobre o tema?

Pretende-se com este artigo auxiliar os praticantes (gestores de pequenas empresas de serviços) no entendimento da necessidade da prática da Gestão Estratégica em suas organizações, sabendo-se que carecem de subsídios teóricos para guiar os seus negócios, assim como para a academia, interessada em consolidar o estado da arte sobre o tema proposto.

Este texto está organizado em seis seções. Após esta breve introdução, será apresentado o objetivo do estudo. Em seguida, serão descritos os procedimentos teórico-metodológicos da pesquisa e, na seção posterior, os principais resultados encontrados, apresentados na seção de Revisão Bibliográfica. Para encerrar o texto, são realizadas as considerações finais do estudo.

\subsection{Objetivo}

Este artigo tem por objetivo realizar uma revisão bibliográfica em torno do conceito Gestão Estratégica, para identificar como as pequenas empresas prestadoras de serviços implementam ações para suportar a ação estratégica.

\section{PROCEDIMENTOSTEÓRICO-METODOLÓGICOS}

Este artigo foi elaborado por intermédio de uma pesquisa e análise bibliométrica, mediante procedimentos de pesquisa quantitativos e qualitativos.

O termo bibliometria, conforme Reveles e Takahashi (2007), é utilizado para quantificar os processos de comunicação escrita, e o emprego de indicadores bibliométricos é útil para medir a produção científica, analisando e avaliando as fontes dos trabalhos, a evolução cronológica da produção científica dos autores e das instituições.

O método quantitativo, segundo Richardson (2008), caracteriza-se pelo emprego da quantificação tanto nas modalidades de coleta de informações, quanto no tratamento delas por meio de técnicas estatísticas. Representa a intenção de garantir a precisão de resultados e é frequentemente aplicado em estudos descritivos para descobrir e classificar a relação entre variáveis que investigam a relação de causualidade entre fenômenos. Tal pesquisa propõe-se a investigar o motivo do fenômeno estudado e deve ser realizada quando o pesquisador deseja obter melhor entedimento do comportamento de diversos fatores e elementos que influem sobre o fenômeno em questão.

Por sua vez, as estratégias de investigação escolhidas em um projeto qualitativo contribuem para um aprofundamento do fenômeno pesquisado. Esse assunto é estudado por Creswell (2007), destacando algumas características da pesquisa qualitativa como: ocorre em um cenário natural, usa métodos múltiplos que são interativos e humanísticos, é emergente, é fundamentalmente interpretativa, vê os fenômenos sociais holisticamente, reflete sistematicamente sobre quem é o pesquisador na investigação e usa um raciocínio 
complexo multifacetado, interativo e simultâneo. As estratégias de investigação da pesquisa qualitativa concentram-se na coleta, registro de dados, análise e na comunicação dos dados, mas têm origem nas disciplinas e fluem durante todo o processo de pesquisa. De acordo com o mesmo autor, os pesquisadores validam os resultados, usando a confiabilidade para verificar consistência dos padrões de desenvolvimento de temas.

Neste estudo, delimitou-se como fonte de pesquisa a base de dados SciELO - Scientific Electronic Library Online (Biblioteca Científica Eletrônica on-line). 0 modelo "SciELO" é produto da cooperação entre a Fundação de Amparo à Pesquisa do Estado de São Paulo (FAPESP) e o Centro Latino-Americano, relacionando a comunicação científica e os editores científicos.

Sua origem se deu a partir de um projeto-piloto, envolvendo dez periódicos brasileiros de diferentes áreas do conhecimento. Foi desenvolvido com êxito entre março de 1997 e maio de 1998, com o desenvolvimento e a avaliação de uma metodologia adequada para a publicação eletrônica na Internet. Desde junho de 1998, o projeto opera regularmente, incorporando novos títulos de periódicos e expandindo sua operação para outros países. A partir de 2002, o Projeto conta com o apoio do Conselho Nacional de Desenvolvimento Científico e Tecnológico (CNPq).

A pesquisa empreendida compreendeu publicações no período entre 2006 e 2012, buscando-se artigos que contivessem em seus títulos o termo "Gestão Estratégica".

Realizou-se posteriormente uma análise documental dos artigos selecionados, pelos critérios anteriormente descritos. Inicialmente, obteve-se uma amostra de vinte artigos que traziam no seu tema o termo "Estratégia". Em seguida, após a leitura dos títulos, foram eliminados os artigos que não tratavam especificamente do tema da Gestão Estratégica. Os treze artigos remanescentes foram submetidos à leitura de seus respectivos resumos, resultando na amostra de cinco artigos selecionados.

Com base nos cinco artigos, a pesquisa procurou encontrar os autores mais citados sob o tema Gestão Estratégica. Tal procedimento de convergência entre os autores encontra-se nas informações da Tabela 1.

Tabela 1 - Autores mais prolíficos

\begin{tabular}{|l||c|}
\hline \multicolumn{1}{|l|}{ AUTORES } & NÚMERO DE CITAÇŌES \\
\hline Ansoff & 2 \\
\hline \hline Hitt & 2 \\
\hline \hline Jarzabkowski & 2 \\
\hline \hline Mintzberg & 6 \\
\hline \hline Porter & 3 \\
\hline Whittington & 2 \\
\hline
\end{tabular}

Fonte: A autora (2012)

Com base nesse levantamento bibliométrico, este estudo procurou aprofundar a sua investigação, realizando a leitura dos autores citados, com o objetivo de destacar os principais procedimentos inerentes à Gestão Estratégica para pequenas empresas de serviços.

\section{REVISÃO BIBLIOGRÁFICA}

Segundo Porter (2009), estratégia é a compatibilização mútua das atividades da empresa, e seu sucesso depende do desempenho positivo de suas atividades e da integração entre elas. Se não houver compatibilidade entre as atividades, não existirá uma estratégia diferenciada e muito menos sustentabilidade.

Conforme os estudos de Mintzberg et al. (2006), a palavra "Estratégia" há tempos vem sendo usada implicitamente de diferentes maneiras, sendo apresentadas pelos autores cinco definições de estratégia conhecidos como os Ps da Estratégia - plano, pretexto, padrão, posição e perspectiva, como segue:

a) Estratégia é um plano, uma diretriz para lidar com uma situação, algum tipo de ação consciente 
pretendida.

b) Estratégia também pode ser um pretexto quando é uma manobra específica para superar um oponente ou concorrente.

c) Estratégia é um padrão em uma corrente de ações, sendo assim realizadas.

d) Estratégia é uma posição, um meio de localizar uma organização em um ambiente.

e) Estratégia como perspectiva é quando seu conteúdo consiste não apenas de uma posição escolhida, mas também de uma maneira fixa de olhar o mundo.

Destaca-se, assim, a importância de inter-relacionamento sobre os Ps da Estratégia, que na prática poderão resultar no processo de Gestão Estratégica.

“Estratégia é um conjunto de objetivos, políticas e planos que define o escopo da empresa e seus métodos de sobrevivência e sucesso" (MINTZBERG et al., 2006, p. 85).

0 trabalho do empreendedor que age estrategicamente deve basear-se em compreender e enfrentar a competição. Conforme Porter (2009), a rivalidade ampliada, que resulta nas cinco forças da estrutura setorial, deve ser analisada e molda a natureza das interações competitivas dentro de um segmento.

"Gestão Estratégia se refere à maneira como as organizações usam graus de liberdade para manobrar entre seus ambientes" (MINZTBERG etal., 2006, p. 41).

As cinco forças estudadas por Porter (2009) orientam os gestores a analisarem o macro ambiente que rodeia as organizações e norteia as tomadas de decisões para o processo de Gestão Estratégica. Destacam-se tais forças:

a) A ameaça de novos entrantes.

b) 0 poder dos fornecedores.

c) $O$ poder dos clientes.

d) A ameaça de substitutos.

e) A rivalidade entre os atuais concorrentes.

A análise das cinco forças deve revelar grandes diferenças dentro de setores muito amplos, indicando a necessidade de ajustar as fronteiras setoriais ou as estratégias decorrentes.

De acordo com Jarzabkowski e Spee (2009), a estratégia como prática preocupa-se com o fazer da estratégia: quem o faz, o que fazem, como fazem ou executam, o que usam e quais implicações tais práticas têm para a formulação de estratégia e para posterior execução da Gestão Estratégica.

Conforme os autores citados, a estratégia está em conformidade com a teoria; entretanto, apenas será válida se utilizável na prática e com o envolvimento dos indivíduos, principalmente dos gestores que devem agir estrategicamente.

Mintzberg etal. (2006, p. 29) definem que

\begin{abstract}
uma estratégia é o padrão ou plano que integra as principais metas, políticas e sequências de ação da organização em um todo coeso. Uma estratégia bem formulada ajuda a organizar e colocar os recursos de uma organização em uma postura única e viável, baseada em suas competências e deficiências internas relativas, mudanças antecipadas no ambiente e movimentos contingentes por parte dos oponentes inteligentes.
\end{abstract}

As empresas devem ser flexíveis para reagir com rapidez às mudanças competitivas e de mercado. Porter (2009) ressalta que o posicionamento estratégico já foi o cerne da estratégia; de acordo com novos dogmas, a vantagem competitiva é uma das melhores hipóteses para a Gestão Estratégica e a competição.

Ainda de acordo com o autor citado, "uma empresa só é capaz de superar em desempenho os concorrentes se conseguir estabelecer uma diferença preservável” (PORTER, 2009, p. 38). 0 posicionamento estratégico significa desempenhar atividades diferentes pelos rivais ou desempenhar as mesmas atividades de maneira diferente.

Tomando a perspectiva da estratégia como prática conceituada por Jarzabkowski (2003), entende-se como uma atividade socialmente realizada, construída por meio de ações, interações e negociações entre atores diversos.

Hitt, Ireland e Hoskisson (2008) destacam que, quando uma capacidade estratégica é valiosa, rara, custosa para imitar e insubstituível, ela é uma competência essencial e torna-se uma vantagem competitiva na Gestão Estratégica de uma empresa.

Como pode a organização existente e a teoria social interagirem na análise da estratégia como prática? De 
acordo com Jarzabkowski (2003), estratégia como prática é a base teórica da estratégia e, como isso, alinha-se com a organização existente e abordagens da teoria social, explicando como fazem e por que fazem a estratégia, e as consequências sociais ao realizar a estratégia como prática.

"Para todo gerente, o processo de elaboração de estratégia começa com uma escolha estratégica fundamental: a descrição teórica da atividade e do ambiente humanos que se encaixam melhor com a própria visão do mundo" (WHITTINGTON, 2006, p. 140).

Porter (2009) destaca que o posicionamento baseado na variedade faz sentido econômico quando a empresa é capaz de produzir produtos e/ou serviços utilizando um grupo de atividades diferenciadas.

A estrutura das decisões da empresa é conceituada por Ansoff (1990) como um modelo de tomada de decisões estratégicas por intermédio de um sistema prático de objetivos, utilizando perfis de sinergia e potencialidades da organização, por meio do processo da Gestão Estratégica. 0 conceito de estratégia situa-se dentro dos focos diversificação e internacionalização, por meio da análise da competitividade das empresas. A aplicação da estratégia dependerá da necessidade do potencial empreendedor, do planejamento de transformação da postura estratégica e do diagnóstico do potencial interno e externo da organização mediante a Gestão Estratégica.

A estratégia não pode ser formulada nem ajustada para circunstâncias mutantes sem um processo de avaliação da estratégia (MINTZBERG et al., 2006).

De acordo com Mintzberg et al. (2006), a estratégia corporativa é o modelo de decisões de uma empresa que determina e revela seus objetivos, propósitos ou metas, produz as principais políticas e planos para atingir essas metas e define o escopo de negócios que a empresa adotará. A decisão estratégica que contribui para esse modelo é eficaz por longos períodos de tempo, afeta a empresa de diferentes formas.

A estratégia corporativa, segundo Mintzberg et al. (2006), é um processo organizacional, que compromete as pequenas empresas prestadoras de serviços, de muitas formas inseparáveis da estrutura, do comportamento e da cultura da empresa onde ocorre. Existem dois aspectos importantes inter-relacionados com o processo organizacional: a formulação da estratégia e a implementação da estratégia:

a) A formulação da estratégia inclui a identificação de oportunidades e ameaças no ambiente externo, e inclusão de algumas estimativas ou riscos para as alternativas discerníveis. Na continuidade desta etapa, é necessário também avaliar as forças e as fraquezas de uma empresa, além dos recursos à mão e disponíveis.

b) A implementação da estratégia compreende um série de subatividades. Se o objetivo é determinado, então podemos mobilizar os recursos de uma empresa para atingi-lo. 0 momento de agir, de criar ações que coloquem na prática os planos criados, entendendo-se assim o processo da Gestão Estratégica com referência aos planos e ações da administração.

Hitt, Ireland e Hoskisson (2008) lembram que as pequenas empresas prestadoras de serviços precisam utilizar ações estratégicas e táticas quando formam suas ações e respostas competitivas no processo da rivalidade competitiva. Uma ação competitiva é uma medida estratégica que a empresa toma para criar ou defender suas vantagens competitivas ou melhorar sua posição no mercado. Uma ação ou resposta estratégica é uma ação baseada no mercado que envolve um comprometimento dos recursos organizacionais aplicados pela Gestão Estratégica.

Conforme os autores citados, os gestores estratégicos são responsáveis por gerenciar o conjunto de estratégias da empresa, coordenam as atividades, categorizam os conhecimentos aprendidos com experiências anteriores e se certificam de que o saber da empresa sobre como formar e usar as estratégias de forma eficaz esteja nas mãos de pessoas certas e no momento certo.

Segundo Mintzberg et al. (2006), para muitas pequenas empresas prestadoras de serviços, a avaliação estratégica é simplesmente uma avaliação de desempenho da empresa, mas, apesar dessa simplicidade, essa linha de raciocínio perde a totalidade do ponto da estratégia - de que os fatores críticos que determinam a qualidade dos resultados atuais nem sempre são diretamente observáveis ou simples de mensurar, e que, quando oportunidades ou ameaças afetarem os resultados operacionais, poderá ser tarde demais para uma resposta eficaz.

Ainda conforme Mintzberg et al. (2006), cada estratégia de negócios é única, seja para grandes, médias ou pequenas empresas; seja para indústrias, comércio ou prestadoras de serviços. A estratégia está principalmente relacionada com a seleção de metas e objetivos, e muitas empresas consideram mais fácil estabelecer ou tentar atingir metas do que as analisar para assim tomar decisões.

Mintzberg et al. ( 2006) observam que o objetivo estratégico é um processo de Gestão Estratégica que 
inclui: focar a atenção da organização na essência da vitória, motivar as pessoas ao comunicar o valor da meta, deixar espaço para contribuições individuais e em equipes, sustentar o entusiasmo fornecendo definições para novas operações à medida que as circunstâncias mudam e usar consistentemente o objetivo de guiar as alocações de recursos.

Segundo Hitt, Ireland e Hoskisson (2008), uma gestão estratégia eficaz das competências essenciais requer a avaliação detalhada dos recursos e das capacitações, recursos que foram deliberadamente integrados para a execução de uma determinada tarefa ou conjunto de tarefas da empresa.

De acordo com os autores mencionados, outro conceito necessário para uma Gestão Estratégica ideal é o empreendedorismo estratégico, que significa tomar ações empreendedoras utilizando uma perspectiva estratégica. As empresas que se decidam ao empreendedorismo estratégico se veem envolvidas simultaneamente em comportamentos de busca por oportunidades e por vantagens que regem a Gestão Estratégica. 0 propósito de agir dessa forma é de encontrar continuamente novas oportunidades e desenvolver inovações para tirar vantagem destas.

Whittington (2006) sugere que o problema para a estratégia é que as organizações, independentemente de seu porte, não pensam e não têm unidade. No entanto, a noção de estratégia implica que todos os indivíduos que compõem uma organização possam unir-se em torno da busca efetiva de meta coerente, compreendendo a necessidade da Gestão Estratégica.

\section{CONSIDERAÇÕES}

Conclui-se com este estudo que a Gestão Estratégica é um processo continuado de tomada de decisões, levando em consideração em primeiro lugar o objetivo estratégico da pequena empresa prestadora de serviços, para posteriormente analisar e conhecer o mais profundo possível os fatores internos - forças e fraquezas - e os fatores externos - oportunidades e ameaças que norteiam a existência de tal empresa.

As organizações precisam pensar e criar as alternativas estratégicas que deverão vir ao encontro com as informações coletadas no diagnóstico estratégico. Posteriormente, toma-se a decisão da melhor alternativa estudada e parte-se para a definição das ações estratégicas desenhadas e organizadas mediante o planejamento estratégico, dividindo-se as ações em planos táticos e operacionais englobando toda a necessidade, levando as empresas à execução por intermédio da Gestão Estratégica.

Por fim, chega-se ao controle estratégico, que com o feedback mostrará o alcance ou não, total ou parcial do objetivo estratégico determinado no início do processo. Caso o objetivo estratégico não tenha sido alcançado, torna-se necessário o novo estudo para diferentes ações e a construção de um planejamento para ações que alterem o processo de Gestão Estratégica.

A principal consideração deste artigo é que todos os autores estudados referenciam em seus estudos a necessidade da utilização da Gestão Estratégica para o sucesso das organizações, o que torna essencial hoje a sua aplicação independentemente do tamanho ou da estrutura das empresas, comprovando, desse modo, a possibilidade de utilização nas pequenas empresas prestadoras de serviço. A autora do artigo defende esta ferramenta em especial nas pequenas empresas prestadoras de serviço, por acreditar que em pequenas corporações a Gestão Estratégica transforma as empresas e é de fácil entendimento, aplicação e de ótimos resultados. 


\title{
STUDY ANALYSIS OF STRATEGIC MANAGEMENT OF SMALL BUSINESS PROVIDERS: a literature review
}

\begin{abstract}
Small service companies have played an increasingly important role in the growth of Brazilian economy. Such companies face new market situations such as competition, excellence in quality, knowledgeable and demanding customers, need to reach the market, culture and organizational climate, suppliers and many other variables are often not known by their administrators. The approach of Strategic Management presents the need to understand the variables and factors that make up the micro and macro environment of small businesses, the planning process and strategic actions that lead to the objectives and goals contributing to the success of companies on a competitive market The aim of this paper is to review literature on the Strategic Management concept, to identify how small service providers implement actions to support the strategic action. The research method was a bibliometric analysis through procedures for qualitative and quantitative research with a documental analysis of publications in the period from 2006 to 2012, searching for articles that contained in their titles the term "Strategic Management" based on data SciELO electronic library.
\end{abstract}

Keywords: Strategic Management. Small business. Literature review.

\section{REFERÊNCIAS}

ALMEIDA, Martinho Isnard Ribeiro de. Modelo de desenvolvimento de planejamento estratégico para pequenas empresas. [2011]. Disponível em:

<http://www.ead.fea.usp.br/eadonline/grupodepesquisa/publica\%C3\%A7\%C3\%B5es/martinho.htm>. Acesso em: 13 ago. 2012.

ANSOFF, Igor H. A nova estratégia empresarial. São Paulo: Atlas, 1990.

CRESWELL, John W. Projeto de pesquisa: métodos qualitativo, quantitativo e misto. 2. ed. Porto Alegre: Artmed, 2007.

HITT, Michael A.; IRELAND, R. Duane; HOSKISSON, Robert E. Strategic management: competitiveness and globalization. 2. ed. São Paulo: Cengage Learning, 2008.

JARZABKOWSKI, Paula. Strategic practices: an activity theory perspective on continuity and change. Journal of Management Studies, v. 40, n. 1, p. 23-55, 2003.

JARZABKOWSKI, Paula; SPEE, Andreas Paul. Strategy-as-pratice: A review and future directions for the Field. International Journal of Management Reviews. Oxford, v. 11, n. 1, p. 69-95, 2009.

MINTZBERG, Henry et al. 0 processo da estratégia: conceitos, contextos e casos selecionados. 4. ed. Porto Alegre: Bookman, 2006.

PORTER, Michael. Competição. Rio de Janeiro: Elsevier, 2009.

REVELES, Audrey Garcia; TAKAHASHI, Regina Toshie. Educação em saúde: um estudo bibliométrico. Rev. esc. enferm. USP [on-line], v. 41, n. 2, 2007.

RICHARDSON, Roberto Jarry. Pesquisa social: métodos e técnicas. 3. ed. São Paulo: Atlas, 2008.

WHITTINGTON, Richard. 0 que é estratégia. São Paulo: Thomson Learning, 2006. 